\title{
Ajustes da Pressão Positiva Expiratória Final Ideal na Síndrome do Desconforto Respiratório Agudo na Posição Prona*
}

\author{
Best Positive end Expiratory Pressure Settlement in Acute \\ Respiratory Discomfort Syndrome and Prone Position
}

\author{
Luiz Rogério de Carvalho Oliveira1, Taciana Gaido Garcia², Vanessa Guimaiel Peres², \\ Kelianne Mayumi Maeda², Juliana Vieira de Oliveira², Juliana Pelissoni Araújo², \\ Camila Vitelli Molinari', Elaine Cristina Poletti', Anderson José3 ${ }^{3}$, Paulo Antônio Chiavone ${ }^{4}$
}

\section{RESUMO}

JUSTIFICATIVA E OBJETIVOS: A posição em decúbito ventral (posição prona) aplicada em pacientes com síndrome do desconforto respiratório agudo (SDRA), tem demonstrado melhora da oxigenação em mais de $70 \%$ dos casos. Uma vez que essa posição promove uma série de alterações pulmonares, inclusive na mecânica pulmonar, abre-se a hipótese da necessidade de otimizar os parâmetros ventilatórios após a instalação deste novo decúbito, principalmente do valor da pressão positiva expiratória final (PEEP). O objetivo deste estudo foi avaliar a influência da posição prona no cálculo da PEEP ideal, titulada pela melhor complacência pulmonar e comparar as alterações pulmonares de mecânica, de oxigenação e de ventilação nas posições supina e prona.

1. Fisioterapeuta; Supervisor do Curso de Especialização em Fisioterapia Respiratória e Fisioterapia em Terapia Intensiva da ISCMSP.

2. Fisioterapeuta; Supervisor do Curso de Especialização em Fisioterapia Respiratória da ISCMSP. .

3. Fisioterapeuta; Professor do Curso de Fisioterapia da UNINOVE. 4. Médico; Doutor em Medicina, Professor Adjunto do Curso de Medicina da FCMSCSP, Diretor do Serviço de Terapia Intensiva da ISCMSP.

*Recebido da Irmandade Santa Casa de Misericórdia de São Paulo (ISCMSP) - Serviço de Reabilitação - Serviço de Terapia Intensiva, São Paulo, SP

Apresentado em 12 de novembro de 2007

Aceito para publicação em 18 de dezembro de 2007

Endereço para correspondência:

Luiz Rogério de Carvalho Oliveira

Rua Osias Dias, $n^{\circ} 174$ - Mairiporã

07600-000 São Paulo, SP

Fone: (11) 4485-4279 ou 6239-3007

E-mail: luizrogerio_oliveira@msn.com

(C)Associação de Medicina Intensiva Brasileira, 2008
MÉTODO: Estudo prospectivo realizado no Serviço de Terapia Intensiva da Irmandade Santa Casa de Misericórdia de São Paulo. Foram comparadas três fases. Fase 1: na posição supina, após o cálculo de PEEP ideal. Fase 2: após duas horas o decúbito do paciente era modificado para prona, após cálculo da PEEP. O paciente era mantido por seis horas neste decúbito. Fase 3: após este período, era colocado na posição supina, realizando-se novo cálculo da PEEP e nova coleta gasométrica, após duas horas. Foi comparada a fase 1 versus fase 2, fase 2 versus fase 3 e fase 3 versus fase 1.

RESULTADOS: Não foram encontradas diferenças nos valores de PEEP ideal nas três fases do estudo: fase 1 $=14 \pm 4,43$, fase $2=14,73 \pm 4,77$ e fase $3=13,65 \pm$ 4,92 .

CONCLUSÕES: Não houve diferença de PEEP ideal quando na posição prona ou supina. Portanto, de acordo com este estudo não há necessidade de se readequar a PEEP a cada mudança de decúbito.

Unitermos: posição prona, PEEP ideal, SDRA, ventilação mecânica

\section{SUMMARY}

BACKGROUND AND OBJECTIVES: In acute respiratory discomfort syndrome (ARDS) patients, prone position improves oxygenation in more than $70 \%$ of the cases. It is well known that prone position promotes a lot of pulmonary changes, including pulmonary mechanics, so we hypothesized that there is the necessity to optimize the ventilatory parameters after the patient is placed in prone position, especially the positive end expiratory pressure (PEEP) values. The objective of this study valued the influence of the prone position at the calculation of the ideal PEEP, given a title by the best pulmonary complaisance and he compared the pulmo- 
nary alterations of mechanics, of oxygenation and of ventilation in the positions supine and prone.

METHODS: Prospective study, taken place in the Irmandade Santa Casa de Misericórdia de São Paulo Intensive Care Service. Three fases have been compared. Fase 1: in supine position, after the best PEEP calculation. Fase 2: two hours after the patient was placed in prone position and the best PEEP was calculated. The patient was kept for 6 hours in this position. Fase 3: after this time, patient was placed in supine again and after two hours, a new best PEEP calculation and arterial gas analysis was done. And then fase 1 versus fase 2 , fase 2 versus fase 3 , fase 3 versus fase 1 were compared.

RESULTS: There were no differences in the PEEP values found in all study fases: fase $1=14 \pm 4$.43; fase 2 $=14.73 \pm 4.77$ and fase $3=13.65 \pm 4.92$.

CONCLUSIONS: There were no differences in best PEEP values between prone and supine position. Therefore, there is no need to recalculate the PEEP value after each position change.

Key Words: ARDS, best PEEP, mechanical ventilation, prone position

\section{INTRODUÇÃO}

A primeira descrição da síndrome do desconforto respiratório agudo (SDRA) foi publicada em 1967 por Ashbaugh e col. Relatava pacientes que apresentavam quadro de insuficiência respiratória atípica, com elevada taxa de mortalidade, diminuição da complacência pulmonar, infiltrados difusos à radiografia de tórax e hipoxemia refratária à administração de oxigênio ${ }^{1-4}$.

Até hoje, essa síndrome vem sendo estudada em seus mais diversos aspectos, Na procura de oferecer estratégias de ventilação mecânica que buscam manter a ventilação e a oxigenação necessárias para a sobrevivência destes pacientes, além de diminuir a lesão pulmonar ocasionada pela pressão positiva ${ }^{2-20}$.

A posição em decúbito ventral (posição prona) aplicada em pacientes com SDRA tem demonstrado melhora da oxigenação em mais de $70 \%$ dos casos, além da melhora da heterogeneidade parenquimatosa, da complacência pulmonar, da relação ventilação-perfusão $V / Q$, diminuição do shunt intrapulmonar, melhora da vasoconstrição pulmonar, do recrutamento alveolar de áreas anteriormente dependentes da gravidade e redução da lesão pulmonar induzida pela ventilação mecânica. Contudo, apesar de tais benefícios, ainda não há evidências concretas que demonstrem diminuição da mortalidade como conseqüência deste posicionamento $3,14,21-27$.

Estudo em animais sugeriu que a ventilação durante a posição prona associada à otimização dos parâmetros ventilatórios, através da análise da mecânica pulmonar, torna mais eficaz o suporte ventilatório, porém levando a necessidade de alterações dos parâmetros ventilatórios ${ }^{28}$.

Uma vez que a posição prona promove uma série de alterações pulmonares, inclusive na mecânica pulmonar, abre-se a hipótese da necessidade de otimizar os parâmetros ventilatórios após a instalação deste novo decúbito, principalmente do valor da pressão positiva expiratória final (PEEP). Assim, diversos estudos apontaram mudanças pulmonares quando a posição prona é adotada. No entanto, as influências destas mudanças nos cálculos de um valor ideal de PEEP, não está bem descrito na literatura.

O objetivo deste estudo foi avaliar a influência da posição prona no cálculo da PEEP ideal, titulada pela meIhor complacência pulmonar e comparar as alterações pulmonares de mecânica, de oxigenação e de ventilação nas posições supina e prona.

\section{MÉTODO}

Após a aprovação pelo Comitê de Ética em Pesquisa da Instituição, foi realizado um estudo prospectivo no Serviço de Terapia Intensiva da Irmandade Santa Casa de Misericórdia de São Paulo.

Foram incluídos os pacientes sedados com diagnóstico de SDRA, caracterizada pela instalação aguda do quadro de insuficiência respiratória, infiltrados difusos à radiografia de tórax, relação $\mathrm{PaO}_{2} / \mathrm{FiO}_{2}$ menor que 200 e pressão de capilar pulmonar $<18$ $\mathrm{mmHg}$ e/ou ausência de sinais de hipertensão atrial esquerda.

Foram excluídos os pacientes com edema cerebral ou hipertensão intracraniana, instabilidades medular e hemodinâmica. Pacientes que necessitaram de retorno emergencial à posição supina, devido a instabilidade hemodinâmica, parada cardiorrespiratória ou necessidade de procedimento invasivo de urgência.

O protocolo iniciava-se com uma fase de estabilização, onde os pacientes eram ventilados mecanicamente na modalidade pressão controlada (PCV), com volumecorrente $(\mathrm{Vt})$ entre 4 e $6 \mathrm{~mL} / \mathrm{kg}$, pressão de platô $<35$ $\mathrm{cmH}_{2} \mathrm{O}, \mathrm{FiO}_{2}$, suficiente para manter a $\mathrm{SaO}_{2}>90 \%, \mathrm{f}$ entre 12 e $20 \mathrm{ipm}$.

- Fase 1: na posição supina, o paciente era submeti- 


\section{AJUSTES DA PRESSÃO POSITIVA EXPIRATÓRIA FINAL IDEAL NA SÍNDROME \\ DO DESCONFORTO RESPIRATÓRIO AGUDO NA POSIÇÃO PRONA}

do à manobra de recrutamento alveolar (MRA) em PCV com pressão inspiratória de $45 \mathrm{cmH}_{2} \mathrm{O}$ e PEEP de 30 $\mathrm{CmH}_{2} \mathrm{O}$ por um minuto; e, cálculo de PEEP ideal, através do método PEEP versus complacência de forma decremental, segundo Suter e col. ${ }^{7}$, seguida de coleta de gasometria arterial duas horas após este procedimento;

- Fase 2: concluída a fase 1 o paciente era colocado na posição prona. Aguardava-se duas horas para estabilização e realizava-se nova manobra de recrutamento alveolar, novo cálculo da PEEP ideal através da complacência e nova análise gasométrica. $O$ paciente era mantido por seis horas neste decúbito, concluindo a fase 2;

- Fase 3: após este período retornava-se à posição supina, feita nova MRA, novo cálculo da PEEP e nova coleta gasométrica após duas horas.

Os parâmetros ventilatórios, a monitorização ventilatória e a análise gasométrica foram comparados entre as três fases do estudo. Além disso, foram correlacionados os dados entre volume-corrente, complacência estática (Cest), $\mathrm{pH}, \mathrm{PaCO}_{2}$ e relação $\mathrm{PaO}_{2} / \mathrm{FiO}_{2}$ com letalidade.

Tabela 1 - Característica da Amostra da População Estudada $(\mathrm{n}=17)$

\begin{tabular}{lcc}
\hline Variáveis & Média & Desvio-Padrão \\
\hline Idade (anos) & 32 & 13 \\
APACHE II (pontos) & 25 & 5 \\
Risco de óbito (\%) & 65 & 15 \\
LIS (Lung Injury Score) & 3,5 & 0,4 \\
\hline
\end{tabular}

A análise estatística foi realizada através dos testes de Kolmogorov-Smirnov(a), Shapiro- Wilk para verificar a distribuição da amostra, testes de Friedman e Wilcoxon para análise das variáveis, estabelecendo o valor de $p$ $<0,05$ para valores estatisticamente significativos.

\section{RESULTADOS}

Ao analisar a distribuição da amostra, verificou-se que não houve distribuição normal, exceto para as variáveis: $\mathrm{pH}, \mathrm{BE}, \mathrm{PaO}_{2}, \mathrm{FiO}_{2}, \mathrm{PaO}_{2} / \mathrm{FiO}_{2}$ e Cest.

Foram avaliados 21 pacientes durante o período compreendido entre março de 2003 e janeiro de 2006. Foram excluídos do estudo quatro pacientes: dois por instabilidade hemodinâmica e dois por apresentarem parada cardiorrespiratória durante o protocolo, tendo seu retorno à posição supina.

A média de idade foi de $32 \pm 13$ anos, o APACHE II foi de $25 \pm 5$ e o risco de óbito de $56 \% \pm 15 \%$ (Tabela 1 ).

A PEEP ideal calculada nas três fases: média de 14 $\pm 4,43$ na fase $1 ; 14,73 \pm 4,77$ na fase 2 ; e, 13,65 \pm 4,92 na fase 3 , todos sem diferença estatística quando comparados entre si $(p=0,603)$.

A $\mathrm{PaO}_{2} / \mathrm{FiO}_{2}$ foi de $135,60 \pm 56,59$ sem diferença estatística em comparação a fase 2: 191,12 \pm 102 ,68, porem com elevação na fase 3: 205,09 \pm 98,1 e diferença quando comparado a fase $1(p=0,006)$.

Não foi encontrada diferença estatística no valor do volume-corrente exalado nas três fases do estudo: fase 1 $=420,92 \pm 161,31$, fase $2=449,31 \pm 132,05$ e fase $3=$ $423,67 \pm 124,39$.

Tabela 2 - Medidas, Resumo e Comparações nas Três Fases

\begin{tabular}{|c|c|c|c|c|c|c|c|c|c|c|}
\hline \multirow[b]{2}{*}{ Variáveis } & \multicolumn{3}{|c|}{ Fase 1} & \multicolumn{3}{|c|}{ Fase 2} & \multicolumn{3}{|c|}{ Fase 3} & \multirow[b]{2}{*}{$p$} \\
\hline & $1^{\circ}$ Quartil & Mediana & $3^{\circ}$ Quartil & $1^{\circ}$ Quartil & Mediana & $3^{\circ}$ Quartil & $1^{\circ}$ Quartil & Mediana & $3^{\circ}$ Quartil & \\
\hline $\mathrm{pH}$ & 7,18 & 7,34 & 7,39 & 7,2 & 7,3 & 7,37 & 7,29 & 7,37 & 7,41 & $p=0,026^{\star}$ \\
\hline $\mathrm{PaCO}_{2}$ & 35,15 & 41,9 & 59,5 & 32,5 & 41 & 56,45 & 35,5 & 42 & 50 & $p=0,128$ \\
\hline $\mathrm{HCO}_{3}$ & 19,7 & 23 & 26,5 & 17,6 & 22 & 27 & 19,85 & 24 & 25,2 & $p=0,919$ \\
\hline $\mathrm{BE}$ & $-4,5$ & -3 & 4,7 & -5 & -1 & 7,95 & $-5,5$ & 0,4 & 5,5 & $p=0,176$ \\
\hline $\mathrm{PaO}_{2}$ & 58,5 & 79 & 111,5 & 82,5 & 105 & 141 & 79,25 & 90 & 118,5 & $p=0,046^{*}$ \\
\hline $\mathrm{SaO}_{2}$ & 86,5 & 92,4 & 97,5 & 93,25 & 97 & 98,4 & 92,6 & 96 & 98,5 & $p=0,04^{\star}$ \\
\hline Pinsp & 27 & 35 & 35 & 29,5 & 33 & 35 & 27,5 & 35 & 35 & $p=0,994$ \\
\hline PEEP & 11 & 14 & 18 & 12 & 15 & 16 & 10 & 12 & 17,5 & $p=0,603$ \\
\hline$F$ & 13 & 16 & 20 & 13 & 18 & 20 & 12 & 18 & 20 & $p=0,401$ \\
\hline $\mathrm{FiO}_{2}$ & 0,525 & 0,7 & 0,9 & 450 & 0,6 & 0,875 & 0,45 & 0,5 & 0,6 & $\mathrm{p}<000^{*}$ \\
\hline Vt & 290 & 362 & 535 & 355 & 410 & 490 & 355 & 450 & 500 & $p=0,300$ \\
\hline Ve & 4,5 & 6 & 6,6 & 5,3 & 6,8 & 8,3 & 5,55 & 6,8 & 9,05 & $p=0,139$ \\
\hline $\mathrm{PaO}_{2} / \mathrm{FiO}_{2}$ & 89,5 & 137,7 & 172 & 118,75 & 158 & 266,15 & 122 & 190 & 260 & $p=0,048^{*}$ \\
\hline Cest & 20,5 & 27,8 & 31 & 25 & 28,57 & 35 & 19,55 & 30 & 37,5 & $p=0,009^{\star}$ \\
\hline
\end{tabular}

Valores expressos em Mediana, $1^{\circ}$ e $3^{\circ}$ Quartis

$\mathrm{p}<0,05$ para dados estatisticamente significativos

Fase 1 = posição supina após ajustes da PEEP; Fase 2 = Posição prona, após ajustes da PEEP; Fase 3 = Posição supina, após ajustes da PEEP 
OLIVEIRA, GARCIA, PERES E COL.

Tabela 3 - Valores das Amostras Colhidas nas Três Fases do Estudo $(n=17)$

\begin{tabular}{|c|c|c|c|c|c|c|}
\hline Variáveis & Fase $1^{*}$ & $\begin{array}{l}\text { Fase } 1 \\
\text { versus } \\
\text { Fase } 2\end{array}$ & Fase 2 * & $\begin{array}{l}\text { Fase } 2 \\
\text { versus } \\
\text { Fase } 3\end{array}$ & Fase 3* & $\begin{array}{l}\text { Fase } 3 \\
\text { versus } \\
\text { Fase } 1\end{array}$ \\
\hline $\mathrm{PH}$ & $7,30 \pm 0,15$ & 0,690 & $7,30 \pm 0,16$ & 0,041 & $7,37 \pm 0,12$ & 0,013 \\
\hline $\mathrm{PaO}_{2}$ & $84,62 \pm 33$ & 0,006 & $112,70 \pm 43,30$ & 0,193 & $95,71 \pm 22,46$ & 0,163 \\
\hline $\mathrm{FiO}_{2}$ & $64,1 \pm 20,70$ & 0,874 & $64,40 \pm 20,00$ & 0,001 & $52,60 \pm 14,30$ & 0,068 \\
\hline $\mathrm{PaO}_{2} / \mathrm{FiO}_{2}$ & $135,60 \pm 56,59$ & 0,076 & $191,12 \pm 102,68$ & 0,679 & $205,09 \pm 98,15$ & 0,006 \\
\hline
\end{tabular}

* Valores expressos em Média \pm DP

Fase 1 = posição supina; Fase 2 = Posição prona, após ajustes da PEEP; Fase 3 = Posição supina, após ajustes da PEEP

Houve elevação da complacência estática de 27,47 $\pm 10,34$ na fase 1 , para $30,69 \pm 10,71$ na fase $2(p=$ 0,003 ); enquanto que quando comparados à fase 3 de $29,84 \pm 11,49$ não mostrou diferença estatística ( $p=$ 0,101 ) (Tabelas 2 e 3).

Ao comparar sobreviventes e não sobreviventes, menores valores de volume-minuto $(p=0,022)$ e de Cest $(p=0,046)$ se correlacionaram com maior mortalidade na fase 1. Na fase 2, a maior letalidade esteve relacionada com a acidose $(p=0,006)$, valores mais baixos de $\mathrm{PaO}_{2}(p=0,041)$ e $\mathrm{SaO}_{2}(p=0,009)$.

\section{DISCUSSÃO}

Grande parte dos estudos sobre a posição prona, relata seu uso sem a alteração nos parâmetros ventilatórios, com o objetivo de se estudar as alterações apresentadas em decorrência apenas do decúbito ${ }^{4,22}$. Contudo, estudou-se a posição prona em conjunto com a estratégia protetora de ventilação mecânica.

Em relação à taxa de mortalidade, os resultados obtidos diferem quando comparados a estudos prévios 3,21-26. A população estudada apresentou mortalidade menor do que a prevista pela média do APACHE II encontrado ( $41 \%$ versus $65 \%)$, mostrando redução de $24 \%$, porém não foi definido neste estudo o método para avaliar e comparar taxas de mortalidade.

A posição prona proporcionou melhora clínica advinda dos efeitos fisiológicos que a técnica propõe, ou seja, melhora da oxigenação, complacência estática, diminuição da $\mathrm{PaCO}_{2}$ com melhora no $\mathrm{pH}$ e aumento da $\mathrm{SaO}_{2}$. A diminuição da $\mathrm{PaCO}_{2}$ vem demonstrando ser um bom indicador de sobrevida dos pacientes com SDRA relacionada à diminuição do espaço morto fisiológico ${ }^{21,22,29,30,34,35}$. Não se pode fomentar esta tese, visto que no presente estudo não houve diminuição da $\mathrm{PaCO}_{2}$.
Observou-se aumento no índice de oxigenação durante a posição prona com média de $135,60 \pm 56,59$ na fase 1 para 205,09 $\pm 98,15$ na fase 3 em seu retorno na posição supina. Isso pode ter ocorrido pelo recrutamento alveolar ocasionado pela posição prona, mesmo sem ter havido alterações nos valores da PEEP ideal, que podem ter sido mascarados pela pequena amostra; no entanto, acredita-se que esta diferença entre as fases perpetue-se na seqüência dos dias, derivada da manobra de recrutamento alveolar e da posição prona, Este aumento da oxigenação também foi encontrado no estudo de Gattinoni e col. ${ }^{22}$, porém não realizaram o recrutamento alveolar e nem reavaliaram a PEEP durante a posição prona.

Nesse estudo 22 os autores relacionaram a melhora da oxigenação somente ao fato do paciente ter sido colocado na posição prona por seis horas, todavia após o tempo estipulado no protocolo proposto do mesmo ensaio clínico, quando os pacientes retornaram para a posição supina, não houve manutenção no índice de oxigenação no decorrer dos dias, mostrando perda do recrutamento alveolar, acomodação da posição prona e perda dos efeitos fisiológicos ou mesmo ausência de resposta à posição prona.

Com relação aos parâmetros ventilatórios utilizados nos três momentos da pesquisa (Pinsp, f, $\mathrm{FiO}_{2}$, Vt, e Ve), não foram observadas modificações significativas em nenhuma das fases propostas. Porém, ao relacioná-los isoladamente notou-se que: quanto maior o valor da PEEP e menor volume-corrente, complacência estática, $\mathrm{pH}, \mathrm{PaO}_{2}, \mathrm{e} \mathrm{SaO}_{2}$ maior a mortalidade ${ }^{15,22,34,35-44}$. Os dois pacientes que apresentaram parada cardiorrespiratória foram reanimados na posição prona, após o sucesso das manobras retornaram à posição supina; os dois tiveram alta do serviço de terapia intensiva e hospitalar, os outros dois que cursaram com instabilidade hemodinâmica durante o posicio- 


\section{AJUSTES DA PRESSÃO POSITIVA EXPIRATÓRIA FINAL IDEAL NA SÍNDROME DO DESCONFORTO RESPIRATÓRIO AGUDO NA POSIÇÃO PRONA}

namento, tiveram melhora quando retornaram à posição supina, sem necessidade de tratamento com vasopressores.

$O$ valor da PEEP ideal calculado através do método PEEP complacência não variou nas posições supina e prona neste estudo. Alterações no recrutamento alveolar, inerentes ao posicionamento podem ter contribuído para estes resultados. Não foram encontrados até o momento outros estudos que relacionassem a otimização dos valores de PEEP na posição prona.

Optou-se em realizar o protocolo iniciando na posição supina, pela facilidade de todos os pacientes estarem nesta posição, no entanto se tivessem sido aleatorizados e alguns iniciassem o estudo na posição prona, talvez os resultados pudessem ser diferentes do exposto pela diminuição do efeito carry-over ou de evolução temporal da doença.

Não houve alterações nos valores da PEEP ideal quando a posição do paciente foi modificada de supina para prona; e, posteriormente seu retorno para supina. Portanto, segundo este estudo, não houve necessidade de se readequar a PEEP a cada mudança de decúbito.

Acredita-se que haja necessidade de novos estudos, com maior amostra, desenhados para avaliar a resposta terapêutica da posição prona em conjunto com a manobra de recrutamento alveolar e a estratégia protetora.

\section{REFERÊNCIAS}

01. Ashbaugh DG, Bigelow DB, Petty TL et al - Acute respiratory distress in adults. Lancet, 1967;12:319-323.

02. Amato MB, Barbas CS, Medeiros DM et al - Beneficial effects in the "open lung approach" with low distending pressures in acute respiratory distress syndrome. A prospective randomized study on mechanical ventilation. Am J Respir Crit Care Med, 1995;152:1835-1846.

03. Kopp R, Kuhlen R, Max M et al - Evidence-based medicine in the therapy of the acute respiratory distress syndrome. Intensive Care Med, 2002;28:244-255

04. Bernard GR, Artigas A, Brigham KL et al - The American-European Consensus Conference on ARDS. Definitions, mechanisms, relevant outcomes, and clinical trial coordination. Am J. Respir Crit Care Med, 1994;149:818-824.

05. Barbas CS, Matos GF, Pincelli MP et al - Mechanical ventilation in acute respiratory failure: recruitment and high positive end-expiratory pressure are necessary. Curr Opin Crit Care, 2005;11:18-28.

06. Ware LB, Matthay MA - The acute respiratory distress syndrome. N Eng J Med, 2000;342:1334-1349.

07. Suter PM, Fairley B, Isenberg MD - Optimum end-expiratory pressure in patients with acute pulmonary failure. N Engl J Med, 1975;292:284-289.

08. Dreyfuss D, Saumon G - Ventilator-induced lung injury: lessons from experimental studies. Am J Respir Crit Care Med, 1998;157:294-323.

09. Muscedere JG, Mullen JB, Gan K et al - Tidal ventilation at low airway pressure can augment lung injury. Am J Respir Crit Care Med, 1994;149:1327-1334.

10. The Acute Respiratory Distress Syndrome Network 2000. Ventilation with lower tidal volume as compared with traditional tidal volume for acute lung injury and acute respiratory distress syndrome. N Eng J Med, 2000;342:1301-1308.
11. The National Heart, Lung, and Blood institute ARDS Clinical Trials Network. Higher versus lower positive end expiratory pressures in patients with the acute respiratory distress syndrome. N Eng J Med, 2004;4:327-336.

12. Ranieri VM, Suter PM, Tortorella $\mathrm{C}$ et al - Effect of mechanical ventilation on inflammatory mediators in patients with acute respiratory distress syndrome: a randomized controlled trial. JAMA, 1999;282:54-61.

13. Hubmayr RD - Perspective on lung injury and recruitment: a skeptical look at the opening and collapse story. Am J Respir Crit Care Med, 2002;165:1647-1653.

14. Gattinoni L, Caironi P, Carlesso E - How to ventilated patients with acute lung injury and acute respiratory distress syndrome. Curr Opin Crit Care, 2005;11:69-76.

15. Amato MB, Barbas CS, Medeiros DM et al - Effect of a protective-ventilation strategy on mortality in the acute respiratory distress syndrome. $\mathrm{N}$ Eng J Med, 1998;338:347-354.

16. Lachmann B - Open the lung and keep the lung open. Intensive Care Med, 1992;18:319-321.

17. Grasso $\mathrm{S}$, Mascia $\mathrm{L}$, Del Turco $\mathrm{M}$ et al - Effects on recruiting maneuvers in patients with acute respiratory distress syndrome ventilated with protective ventilatory strategy. Anesthesiology, 2002;96:795-802.

18. Rouby JJ, Lu Q, Goldstein I - Selecting the right level of positive endexpiratory pressure in patients with acute respiratory distress syndrome. Am J Respir Crit Care Med, 2002;165:1182-1186.

19. Tobin MJ - Advances in mechanical ventilation. N Eng J Med, 2001;344:1986-1996.

20. Valenza F, Guglielmi M, Maffioletti M et al - Prone position delays the progression of ventilator-induced lung injury in rats: does lung strain distribution play a role? Crit Care Med, 2005;33:361-367.

21. Paiva KCA, Beppu OS - Posição prona J Bras Pneumol. 2005;31:332-40

22. Gattinoni L, Tognoni G, Pesenti A et al - Effect of prone positioning on the survival of patients with acute respiratory failure. $\mathrm{N}$ Eng J Med, 2001;345:568-573.

23. Messerole E, Peine $\mathrm{P}$, Wittkopp $\mathrm{S}$ et al - The pragmatics of prone positioning. Am J Respir Crit Care Med, 2002;165:1359-1363.

24. Pelosi P, Bottino N, Chiumello D et al - Sigh in supine and prone position during acute respiratory distress syndrome. Am J Respir Crit Care Med, 2003;167:521-527.

25. Staudinger T, Kofler J, Mullner $\mathrm{M}$ et al - Comparison of prone positioning and continuous rotations on patients with adult respiratory distress syndrome: results of a pilot study. Crit Care Med, 2001;29:51-56.

26. Flaatten $\mathrm{H}$, Aardal $\mathrm{S}$, Hevroy $\mathrm{O}$ - Improved oxygenation using the prone position in patients with ARDS. Acta Anaesthesiol Scand, 1998;42:329-334

27. Vollman KM, Bander JJ - Improved oxygenation utilizing a prone position in patients with acute respiratory distress syndrome. Intensive Care Med, 1996;22:1105-1111.

28. Broccard A, Shapiro RS, Schmitz LL et al - Prone positioning attenuates and redistributes ventilator-induced lung injury in dogs. Crit Care Med, 2000;28:295-303

29. Oczenski W, Hormann C, Keller $\mathrm{C}$ et al - Recruitment maneuvers during prone position in patients with acute respiratory distress syndrome. Crit Care Med, 2005;33:54-61.

30. Langer M, Mascheroni D, Marcolin R et al - The prone position in ARDS patients. A clinical study. Chest, 1988;94:103-107.

31. Fridrich $\mathrm{P}, \mathrm{Krafft} \mathrm{P}, \mathrm{Hochleuthner} \mathrm{H}$ et al - The effects of long-term prone positioning in patients with trauma-induced adult respiratory distress syndrome. Anesth Analg, 1996;83:1206-1211.

32. Antoniazzi $P$, Pereira Junior GA, Marson $\mathrm{F}$ et al - Síndrome da angústia respiratória aguda. Medicina Ribeirão Preto. Simpósio: Medicina Intensiva: II. Tópicos Selecionados 1998;31:493-506.

33. Gattinoni L, Vagginelli $\mathrm{F}$, Carlesso $\mathrm{E}$ et al - Decrease in $\mathrm{PaCO}_{2}$ with prone position is predictive of improved outcome in acute respiratory distress syndrome. Crit Care Med, 2003;31:2727-2733.

34. Slutsky AS - The acute respiratory distress syndrome, mechanical ventilation, and the prone position. N Engl J Med, 2001;345:610-612.

35. Grasso S, Mascia L, Del Turco $M$ et al - Effects of recruiting maneuvers in patients with acute respiratory distress syndrome ventilated with protective ventilatory strategy. Anesthesiology, 2002;96:795-802.

36. Pappert $\mathrm{D}$, Rossaint $\mathrm{R}$, Slama $\mathrm{K}$ et al - Influence of positioning on ventilation-perfusion relationships in severe adult respiratory distress syndro- 
me. Chest, 1994;106:1511-1516.

37. Jolliet $\mathrm{P}$, Bulpa $\mathrm{P}$, Ritz $\mathrm{M}$ et al - Additive beneficial effects of the prone position, nitric oxide, and almitrine bismesylate on gas exchange and oxygen transport in acute respiratory distress syndrome. Crit Care Med, 1997;25:786-794.

38. Mure M, Martling CR, LindahI SG - Dramatic effect on oxygenation in patients with severe acute lung insufficiency treated in the prone position. Crit Care Med, 1997;25:1539-1544.

39. Blanch L, Mancebo J, Perez M et al - Short-term effects of prone position in critically ill patients with acute respiratory distress syndrome. Intensive Care Med, 1997;23:1033-1039.

40. Chatte G, Sab JM, Dubois JM et al - Prone position in mechanically ventilated patients with severe acute respiratory failure. Am J Respir Crit
Care Med, 1998:155:473-478.

41. Papazian L, Bregeon F, Gaillat F et al - Respective and combined effects of prone position and inhaled nitric oxide in patients with acute respiratory distress syndrome. Am J Respir Crit Care Med, 1998;157:580-585.

42. Jolliet P, Bulpa P, Chevrolet JC - Effects of prone position on gas exchange and hemodynamics in severe acute respiratory distress syndrome. Crit Care Med, 1998;26:1977-1985.

43. Martinez M, Diaz E, Joseph D et al - Improvement in oxygenation by prone position and nitric oxide in patient with acute respiratory distress syndrome. Intensive Care Med, 1999;25:29-36.

44. Lim CM, Koh Y, Chin JY et al - Respiratory and haemodynamic effects of the prone position at two different levels of PEEP in a canine acute lung injury model. Eur Respir J, 1999:13:163-186. 